\title{
Sprachpuristische Bestrebungen des 19. Jahrhunderts am Beispiel des Verdeutschungswörterbuchs von Johann Gottfried Sommer
}

\section{The 19th Century Linguistic Purism Attempts in Johann Gottfried Sommer's Dictionary}

Eva Janečková

\begin{abstract}
The present study explores a largely forgotten Germanization dictionary published in early decades of the $19^{\text {th }}$ century in Prague. A short overview of J. G. Sommer's life will be presented as well as his set of rules on Germanizing loanwords, and a second edition of his dictionary published in 1819 will be described. The main part of the study comprises of presenting the results of an analysis conducted on two selected parts of the dictionary and a brief comparison between Sommer's work on purism and the work of one the most famous authors of Germanization dictionaries published in Germany, Joachim Heinrich Campe.
\end{abstract}

\section{Keywords}

Johann Gottfried Sommer; linguistic purism; Germanisation; lexicography

Die Verfassung dieses Artikels wurde durch finanzielle Unterstützung vom Ministerium für Schulwesen, Jugend und Sport der Tschechischen Republik im Jahre 2019 ermöglicht, das die Wissenschaftsproduktion an der Philosophischen Fakultät der Palacky-Universität Olomouc fördert (IGA_FF_2019_030). 


\section{Einführung}

Mit den puristischen Bestrebungen, deren Ziel in der Vermeidung der Fremdwörter bzw. Entlehnungen und in ihrer Ersetzung durch indigene Ausdrücke bestand, reagierten die Deutschen auf das in vielen Bereichen häufige Vorkommen dieser Wörter zu Ungunsten ihrer Muttersprache. Zu den bedeutenden Gebersprachen gehörten insbesondere das Lateinische, Französische und Italienische. Das Lateinische galt nicht nur als die Sprache der Geistlichkeit, sondern es spielte auch eine Schlüsselrolle in den Wissenschaften und im Bereich der Juristerei, weil im deutschen Sprachraum das römische Recht rezipiert wurde (vgl. Straßner 1995: 2-35). Die französische Sprache beeinflusste den Wortschatz des Deutschen v. a. in der Kultur. Vom 11. bis 13. Jh. wurden viele Ausdrücke der Hofkultur und des Rittertums entlehnt, z. B. Turnier oder Lanze, und im 17. Jh., d. h. in der Alamodezeit ${ }^{1}$ und während des Dreißigjährigen Krieges, kam es ebenfalls zur Entlehnung vieler französischer Ausdrücke. Außerdem lässt sich der französische Einfluss in der Zeit der Aufklärung beobachten. In der Zeit der Renaissance und des Barock gelangten aus dem Italienischen ins Deutsche die Ausdrücke aus den Bereichen Musik (z. B. Konzert), Bank- und Kaufmannswesen (z. B. Konto, Bankrott) und Militär, wie Soldat oder Kanone (Eisenberg 2011: 57-67).

Die Beschäftigung mit dem Sprachpurismus² fängt mit den ersten deutschen Sprachgesellschaften an, die im 17. Jh. nach italienischen und französischen Vorbildern gegründet wurden (vgl. Haß-Zumkehr 2001: 67). Der Sprachpurismus war nicht nur bei den Sprachgelehrten, zu denen Johann Christoph Adelung, Jacob Grimm oder Philipp von Zesen gehörten, ein relevantes Thema, sondern die Frage nach der Reinheit und Reinigung der Sprache weckte Interesse auch bei anderen Gelehrten, z. B. bei den Schriftstellern Johann Wolfgang von Goethe und Jean Paul oder bei dem Philosophen Gottfried Wilhelm Leibniz ${ }^{3}$ (vgl. Straßner 1995: 75-184). Neben theoretischen Schriften, die sich dem Sprachpurismus widmeten, entstanden Verdeutschungswörterbücher, deren Autoren sich zum Ziel setzten, viele aus anderen Sprachen übernommene Wörter in ihrer Muttersprache zu ersetzen. Viele Verdeutschungswörterbücher entstanden im Laufe des 19. Jh. Zu ihnen gehörte auch das Wörterbuch von Johann Gottfried Sommer, das im Unterschied zu bekannteren Werken außerhalb des zentralen deutschen Sprachraums herausgegeben wurde. Dieses Wörterbuch wird im Folgenden vorgestellt und analysiert.

1 In dieser Zeit wurde das Leben des Adels und des Bürgertums durch französische Einflüsse geprägt, v. a. im gesellschaftlichen und kulturellen Bereich. Die neuen Ausdrücke waren z. B. Tante oder nett (vgl. Eisenberg 2011: 58).

2 Für die Definition des Purismus vgl. z. B. Duden (1997: 674), Fremdwörterbuch (1966: 588) und Deutsches Wörterbuch (1997: 994). Den Termini Sprachpurismus, Fremdwortpurismus und ihren Gemeinsamkeiten und Unterschieden widmen sich Polenz (1994: 107) und Kirkness (1998: 407).

3 Es gab zwei grundsätzliche Stellungnahmen zum Einfluss der Fremdsprachen auf das Deutsche. Den radikaleren Vertretern des Sprachpurismus, die alle oder fast alle Wörter fremden Ursprungs oder alle von Fremdsprachen beeinflussten Ausdrücke vermeiden wollten, wie z. B. Philipp von Zesen oder Joachim Heinrich Campe, standen diejenigen Sprachpfleger gegenüber, die das Vorkommen dieser Wörter nach der Situation und der Textsorte bewerteten, u. a. Gottfried Wilhelm Leibnitz und Johann Christoph Adelung (vgl. Straßner 1995: S. 75-184). 


\section{Johann Gottfried Sommers Verdeutschungswörterbuch}

\subsection{Sommers Leben}

Johann Gottfried Sommer stammte aus dem Dorf Leuben bei Dresden, wo er im Jahre 1782 (1783) geboren wurde. Sein wahrer Name war Volte. Den Großteil seines Lebens war er als Lehrer tätig, zuerst an der Garnisonschule in Dresden und von 1818 bis 1831 als Professor für wissenschaftliche Gegenstände am Konservatorium in Prag, wo er sich niedersetzte und bis zu seinem Tode lebte. Daneben wirkte er als Redakteur der Zeitschriften Hesperus und Oekonomische Neuigkeiten und nahm an der Herausgabe des Taschenbuchs zur Verbreitung geographischer Kenntnisse teil. Dem Bereich der Geographie widmete er sich intensiver und publizierte mehrere geographische Werke, u. a. das Königreich Böhmen, statistisch-topographisch dargestellt, das in den Jahren 1833-1849 erschien. Sein Leben lang beschäftigte er sich auch mit dem Studium von modernen Sprachen. Er starb am 11. November (oder am 12. November) 1848 in Prag (vgl. z. B. NND 1851: 43f. und BLKO 1877: 286-289).

\subsection{Sommers Verdeutschungsprogramm}

Johann Gottfried Sommer gab keine theoretischen Schriften heraus, in denen er sich mit der Verdeutschungsarbeit befasst hätte. Aus diesem Grund können seine Einstellungen zum Sprachpurismus nur aus dem Vorbericht zur ersten Auflage (Sommer 1819, III-XII) und der Vorrede zur zweiten Auflage (Sommer 1819, XIV-XVII) seines Wörterbuchs gewonnen werden. ${ }^{4}$ Zur Verdeutschungsarbeit und zur Verfassung seines Wörterbuchs führten ihn wahrscheinlich seine nationalistischen Ansichten, die sich aus den obengenannten Teilen des Wörterbuchs herauslesen lassen, anhand deren er mit großer Wahrscheinlichkeit zu den radikaleren Vertretern des Sprachpurismus gezählt werden kann. ${ }^{5}$

Bei der Arbeit an seinem Wörterbuch benutzte Sommer mehrere zeitgenössische Verdeutschungswörterbücher, zweisprachige Wörterbücher und weitere Nachschlagewerke. Aus ihnen hebt er u. a. die Verdeutschungsarbeit von Joachim Heinrich Campe ${ }^{6}$ hervor. Aus Sommers Bewertung vieler dieser Wörterbücher lassen sich die Grundregeln seiner Verdeutschungsarbeit schließen. Die idealen Verdeutschungen sind nach ihm einsilbig und sie müssen weitere Wortbildungen ermöglichen. Geeignete Quellen zur Bildung der Verdeutschungen sind nach ihm ältere Stadien der deutschen Sprache, Dialekte und

4 Die späteren Auflagen (1825 und 1833) beinhalten sogar keine Verdeutschungsregeln.

5 Am Ende des Vorberichts zur ersten Auflage steht: „Möchte es [das Wörterbuch] beitragen, die herrliche Sprache unserer Väter von dem entehrenden ihr aufgebürdeten Flitterstaate zu reinigen!" (Sommer 1819: XI). In der Vorrede zur zweiten Auflage schrieb Sommer über die „Vertreibung der Ausländer" (Sommer 1819: XIII). Da die deutschen Gebiete am Ende des 18. und Anfang des 19. Jh. von Franzosen militärisch gefährdet waren, reagierten diese Äußerungen ohne Zweifel auf diese Situation (vgl. Müller 1995, 125-142).

6 Joachim Heinrich Campe verfasste 1801 das Wörterbuch zur Erklärung und Verdeutschung der unserer Sprache aufgedrungenen fremden Ausdrücke, das sehr einflussreich war (vgl. Polenz 1994: 133 ff.). 
Ausdrücke aus anderen germanischen Sprachen. ${ }^{7}$ Für einen großen Mangel hält er die Tatsache, dass viele Verdeutschungswörterbücher sehr oft nur wörtliche Übersetzungen von Fremdwörtern oder Erklärungen in Form von Komposita angeben. ${ }^{8}$

Nach Sommer gibt es Wörter, die in ein Verdeutschungswörterbuch nicht aufgenommen werden sollten. Es handelt sich um Modewörter und um fremdsprachige Ausdrükke, die nur Gelehrten vertraut sind. Im Wörterbuch sollen nur die im Deutschen bereits etablierten Wörter erscheinen. Neben der Auswahl von Lemmata kritisiert er bei anderen Autoren lange Wörterbuchartikel und fordert möglichst sachliche und kurze Einträge. ${ }^{9}$ Da jedoch sein Wörterbuch auch für ungelehrte und ungebildete Menschen ${ }^{10}$, die die Zielgruppe von Lesern darstellen, bestimmt ist, sollten die Artikel gleichzeitig viele Erklärungen beinhalten.

Da Sommer keine anderen Regeln im Metateil seines Wörterbuchs festlegte und keine theoretischen Schriften zu diesem Thema verfasste, kann vorausgesetzt werden, dass er auf andere Bereiche und Probleme, mit denen sich andere Sprachpuristen beschäftigten, nicht einging.

\subsection{Sommers Wörterbuch und seine Struktur}

Die erste Auflage von Sommers Verdeutschungswörterbuch wurde im Jahre 1813 publiziert. Im Folgenden wird die zweite veränderte Auflage analysiert, die im Jahre 1819 in Prag unter dem Titel Neuestes wort- uns sacherklärendes Verteutschungswörterbuch aller jener aus fremden Sprachen entlehnten Wörter, Ausdrücke und Redensarten, welche die Teutschen bis jetzt, in Schriften und Büchern sowohl als in der Umgangssprache, noch immer für unentbehrlich und unersetzlich gehalten haben; verbunden mit einer Erklärung auch der weniger bekannten Kunstwörter und andern Ausdrücke der teutschen Sprache. Ein höchstnützliches Handbuch für Geschäftsmänner, Zeitungsleser und alle gebildete (sic!) Menschen überhaupt (im Weiteren NWVW) herausgegeben wurde. Das Wörterbuch wurde dann noch in den Jahren 1825, 1833 und 1839 veröffentlicht (vgl. das BLKO 1877: 287).

Die zweite Auflage von NWVW hat einen Umfang von etwa 551 Seiten. Der Metateil des Wörterbuchs beinhaltet den Vorbericht zur ersten Auflage und die Vorrede zur zweiten Auflage, die Erklärung der Abkürzungen, die Sommer im Wörterbuchteil verwendet, und am Ende des Wörterbuchs befindet sich noch eine Liste mit Druckfehlern und Verbesserungen. Der Wörterbuchteil erstreckt sich über 532 Seiten. Die Lemmata sind alphabetisch geordnet, aber weil die alphabetische Reihenfolge der Lemmata an manchen Stellen ge-

7 Die angeführten Regeln stützen sich auf seine Bewertung der Verdeutschungsarbeit von Karl Christian Müller und Lorenz Oken.

8 Gleichzeitig lässt er aber zu, dass er im Falle, dass er keine geeignete Verdeutschung fand, auch diese Form der Bedeutungsangabe verwendete.

9 Zur Tatsache, dass auch Campe sehr oft umfangreiche Wörterbuchartikel schrieb, äußert sich Sommer nicht.

10 Im gewissen Widerspruch zu dieser seiner Äußerung steht jedoch der aufwertende Untertitel seines Wörterbuchs: „Ein höchstnützliches Handbuch für Geschäftsmänner, Zeitungsleser und alle gebildete Menschen überhaupt“. 
brochen wird ${ }^{11}$, kann man sie wahrscheinlich nicht als striktalphabetisch bezeichnen. Auf jeder Seite des Wörterbuchs sind die Lemmata in zwei Spalten verteilt und jedes Lemma fängt mit einem Großbuchstaben an, auch wenn es sich nicht um Substantive, sondern um Verben, Adjektive oder andere Wortarten handelt. Die Wörterbuchabschnitte haben immer zwei Teile. Den ersten Teil bilden die Abkürzungen, bei denen ihre Entzifferungen bzw. Äquivalente oder Paraphrasen angeführt werden..$^{12}$ Im zweiten Teil erscheinen die Lemmata, die zu dem konkreten Buchstaben gehören. In diesem Teil befinden sich die vollständigen Formen der Abkürzungen sehr oft noch einmal. Für die Lemmata der Buchstaben I und J gibt es einen gemeinsamen Wörterbuchabschnitt.

Um die im Deutschen bereits etablierten Lexeme von den Fremdwörtern, d. h. noch nicht etablierten Lexemen zu unterscheiden, verwendet Sommer zwei unterschiedliche Schriftarten. Die erstgenannten Wörter wurden in Fraktur gedruckt und die Lexeme, die in die zweitgenannte Gruppe gehören, erscheinen in Antiquaschrift. Diese Unterscheidung findet man sowohl bei Lemmata als auch bei Beispielsätzen und Kollokationen. In den kürzeren Bedeutungsangaben in NWVW erscheint nur eine Bedeutung, während man bei Lemmata, die mehrere Bedeutungen haben, mehrere mit Zahlen voneinander unterschiedene Bedeutungsangaben findet. Die Bedeutungsangaben kommen in Form eines Äquivalents oder einer Paraphrase vor. ${ }^{13}$ Diese Unterscheidungen wurden auch in die im nächsten Kapitel behandelte Analyse einbezogen.

\section{Analyse}

\subsection{Methodologisches}

Diese Analyse verfolgt das Ziel, anhand von zwei ausgewählten Wörterbuchabschnitten erste Informationen über Sommers lexikographische Arbeit zu gewinnen. Der erste Abschnitt beinhaltet 563 Lemmata mit dem Anfangsbuchstaben L, die sich über 15 Seiten erstrecken, und im zweiten analysierten Abschnitt befinden sich 1194 Lemmata auf etwa 28 Seiten. Die Kriterien, nach denen die Lemmata analysiert wurden, betreffen ihre Wortart, adaptierte oder nichtadaptierte Schriftart, ihre Form (Simplex oder Kompositum), semantische Klasse (Autosemantikum oder Synsemantikum), Etymologie und ggf. auch die von Sommer verwendete Zuordnung zu einem Fachgebiet. Des Weiteren wurde bewertet, ob die Wörterbuchartikel Äquivalente oder Paraphrasen von

11 Zum Beispiel beim Lemma Litis consortes, das vor dem Wort Lisbonnine steht, weil es lexikalisch sowie semantisch mit dem Lemma Lis zusammenhängt (S. 296), oder bei der Nominalphrase Etudiant en medecine, die Sommer aus einem unbekannten Grund vor das Lemma Etudiant en droits anordnete (S. 184).

12 Es handelt sich z. B. um die Erklärung „Pfund Vlämisch“ beim Lemma L. vl. (S. 287).

13 Unter dem Begriff Äquivalent versteht man die Ersetzung eines einwortigen oder mehrwortigen Lemmas durch ein deutsches Wort. Die Paraphrase bedeutet eine aus mehreren Wörtern bestehende Bedeutungsangabe. Wenn eine Phrase als Lemma dient und die Bedeutungsangabe durch mehrere Wörter realisiert wird, wird für die Zuordnung zu einer Kategorie der Umfang und die Bedeutung maßgebend. 
Lemmata angeben, und ob Lemmata in anderen Wörterbüchern des 19. Jh. und speziell in Wörterbüchern von Adelung und Grimm ${ }^{14}$ vorkommen.

Im Rahmen der Analyse wurden einige Grundregeln beachtet. Während bei anderen Kriterien die konkreten Zahlenangaben und Prozentanteile festgestellt wurden, wurde wegen des Charakters von Kriterien bei der Einteilung von Lemmata nach der Etymologie und nach ihrem Vorkommen in anderen Wörterbüchern des 19. Jh. und bei Adelung und Grimm ${ }^{15}$ nur eine grobe Anordnung in einzelne Kategorien durchgeführt. Im Falle, dass ein Wörterbuchartikel nur auf einen anderen Artikel bzw. dessen Teil verweist, wurde das zu diesem Artikel gehörte Lemma nicht in die Analyse einbezogen. Bei der Analyse von Lemmata gab es auch Fälle, in denen die Zuordnung zu einer konkreten Kategorie nicht eindeutig war. Wenn mehrere Lemmata zu einem Wörterbuchartikel gehören, dann wird das erste Lemma in die Analyse einbezogen. Weitere Regeln, die für die Analyse von einzelnen Kriterien von Bedeutung sind, erscheinen im folgenden Kapitel bei einzelnen Kriterien.

\subsection{Ergebnisse der Analyse}

Im Rahmen des ersten Kriteriums, d. h. bei der Gliederung nach der Wortart, wurden wegen vieler mehrwortiger Lemmata und wegen Abkürzungen, die im Wörterbuch ebenfalls als Lemmata vorkommen, auch die Kategorien Phrase und Abkürzung unterschieden. Die Substantive stellen die am häufigsten vorkommende Wortart dar und bilden in beiden Wörterbuchabschnitten mehr als $50 \%$ aller Lemmata (z. B. Logomachie, S. 299; Editor, S. 168) ${ }^{16}$ Dieser Wortart folgen Verben (z. B. Evolviren, S. 186; Locupletiren, S. 298) und Adjektive (u. a. Elastisch, S. 169; Lyrisch, S. 301), die beim Buchstaben L etwa $9 \%$ und $8 \%$, bei den Lemmata mit dem Anfangsbuchstaben E dann ungefähr $20 \%$ und $11 \%$ betragen. Beim L gibt es einen höheren Anteil von Phrasen (z. B. Lapis philosophorum, S. 289) und Abkürzungen (L. S., S. 287), während sich beim zweiten Abschnitt eine größere Anzahl verschiedener Kategorien beobachten lässt, neben den bereits genannten auch eine Konjunktion, Präfixe, eine Präposition und Pronomina.

Im Rahmen der Unterscheidung von adaptierten und nichtadaptierten Lemmata ${ }^{17}$ wurden im Falle, dass bei Artikeln sowohl adaptierte als auch nichtadaptierte Lemmata

14 Namentlich sind es das Deutsche Wörterbuch von den Gebrüdern Grimm und das Grammatisch-kritische Wörterbuch der Hochdeutschen Mundart mit beständiger Vergleichung der übrigen Mundarten, besonders aber der oberdeutschen von Johann Christoph Adelung.

15 Grimms Wörterbuch wurde später publiziert und erst in den 60er Jahren des 20. Jahrhunderts vollendet (vgl. Haß-Zumkehr 2001: 119). Aus diesem Grund diente das Werk bei der Analyse v. a. zur Kontrolle.

16 Substantive als Lemmata erscheinen im Wörterbuch manchmal im Plural, z. B. Lamellen (S. 289). Bei diesen Lexemen war der Autor wahrscheinlich der Meinung, dass diese Wörter nur ausnahmsweise im Singular verwendet werden.

17 Die Unterscheidung betrifft in einem Fall auch Komponenten eines Kompositums (Essito-Zoll, S. 183), sodass das Bestimmungswort nicht adaptiert und das Grundwort adaptiert ist. 
erscheinen, diese Artikel zur Gruppe der adaptierten Lexeme gezählt. ${ }^{18}$ Der Prozentanteil von adaptierten Lexemen wie Extenuiren (S. 193) oder Lyra (S. 301) beträgt in beiden Abschnitten mehr als $50 \%$ (beim E mehr als $60 \%$ ). Da es bei einigen Wörterbuchartikeln mehrere orthographische Varianten bei Wörtern gab (z. B. Epilog und Epilogus; S. 180), kann vorausgesetzt werden, dass sie nicht einheitlich verwendet wurden.

Die Markierung des Fachgebiets fehlt bei etwa $80 \%$ aller Artikel. Bei den übrigen Wörterbuchartikeln findet man die Angabe des Fachgebiets entweder für den ganzen Artikel (bei mehr als $80 \%$ aller Lemmata) oder nur für eine der Bedeutungen. Die meisten Lemmata stammen aus dem Bereich Arzneikunde (fast $15 \%$ beim Buchstaben L und etwa $21 \%$ beim E). Es handelt sich z. B. um Lemmata Endeixis (S. 175) und Lepra (S. 293). Dieselbe Anzahl betragen auch die Lemmata mit dem Anfangsbuchstaben L, die von Sommer der Musik zugeordnet wurden, wie Lagrimoso (S. 289) oder Ligato (S. 295). Am zweiten Platz befindet sich die Fachgebietsangabe juristisch, z. B. bei Lemmata Evocatorium (S. 186) und Laesio enormis (S. 288). Im Gegensatz zur Wortstrecke L erscheinen bei Wörtern auf E die Sachgebiete wie Algebra, Arabisch, Baukunst, Grammatik oder Chronologie, im zweiten Abschnitt handelt es sich um die Bereiche Buchdruckerkunst, Fechtkunst, Geographie, Mathematik oder Römische Kirche. ${ }^{19}$

Bei den meisten Lemmata handelt es sich um Simplizia (etwa 70 \% der Lemmata). Für Komposita wurden zum Zwecke dieser Analyse auch diejenigen Lexeme gehalten, die im Deutschen nicht mehr zerlegbar sind, obwohl sie ursprünglich aus mehreren Komponenten zusammengesetzt wurden (z. B. das Lemma Etymologie, S. 184). Die Komposita kombinieren manchmal die fremden und deutschen Komponenten, wie z. B. bei Lexemen Ehepacten (S. 169) und Leibesconstitution (S. 292). Aus dieser Tatsache lässt sich schließen, dass solche Ausdrücke fremden Ursprungs im Deutschen bereits etabliert waren und zur Bildung neuer Wörter benutzt wurden.

In analysierten Wörterbuchabschnitten überwiegen Autosemantika (etwa $75 \%$ bei Buchstaben L und fast 85 \% beim E). Das Ergebnis korreliert mit den Ergebnissen aus der Unterscheidung der Lemmata nach ihrer Wortart, in der Substantive, Verben und Adjektive die am häufigsten vorkommenden Wortarten darstellten. Zu Synsemantika, die weniger als $1 \%$ betragen, gehören z. B. Partikeln, Präpositionen oder Pronomina.

Johann Gottfried Sommer bemühte sich darum, zu den meisten Lemmata statt mehrwortiger Bedeutungsangaben ein einwortiges Äquivalent hinzufügen (vgl. Sommer 1819: S. XVI). Die Äquivalente findet man bei etwa $70 \%$ der Lemmata beim Abschnitt L und bei mehr als $80 \%$ bei Lexemen auf E. Es handelt sich z. B. um die Bedeutungsangabe „ausweichend, vereitelnd“ beim Lemma Elusivisch (S. 171). Zu den Paraphrasen gehört die Bedeutung "päpstlicher Gesandter, der zugleich Cardinal ist" beim Lemma Legatus a latere (S. 292). Es lassen sich zwei Grundtypen von Paraphrasen unterscheiden. Der erste Typ der Paraphrase erklärt die Bedeutung eines Lemmas durch mehrere Wörter und dient dazu, das Wort in einer kommunikativen Situation richtig zu verwenden, z. B. die

18 Die Tatsache, dass es bereits zur Anpassung dieser Wörter an das Deutsche kam (zumindest nach dem Autor), spielt eine bedeutende Rolle.

19 Interessanterweise haben einige Abkürzungen im Wörterbuch eine andere Form als im Abkürzungsverzeichnis. 
Paraphrase „Versuche anstellen“ beim Lexem Experimentiren (S. 191). Die Paraphrasen des zweiten Typs erweitern das enzyklopädische Wissen des Wörterbuchbenutzers, indem sie ihn u. a. über mythologische Figuren (z. B. Eris - „Göttinn des Streites“, S. 182) oder Erfindungen (z. B. Electrometer - „Werkzeug die Stärke der Electricität zu messen“, S. 170) informieren. Neben Äquivalenten und Paraphrasen unterscheidet man im Rahmen dieses Kriteriums auch Entzifferung der Abkürzungen (vgl. Kap. 2. 3., S. 21). Diese Kategorie wird in Wörterbuchartikeln sehr häufig mit Äquivalenten und Paraphrasen kombiniert. In ausgewählten Wörterbuchabschnitten gibt es auch Lehnübersetzungen (z. B. Loco sigilli - „anstatt des Siegels“, S. 298) und Lehnübertragungen (z. B. Lithographie - „Steinbeschreibung“, S. 297), entweder in Form einer Nominalphrase oder eines Kompositums. Viele von ihnen stammen aus dem Lateinischen, Griechischen und Französischen.

Die Etymologie der Lexeme wurde aus anderen damaligen Nachschlagewerken ergänzt. Die größte Anzahl von Lemmata stammt aus dem Lateinischen (etwa $50 \%$ beim L und $30 \%$ beim E). Die meisten von ihnen sind adaptiert und gehören zu den Fachgebieten Arzneikunde, Mythologie oder juristisch. Es handelt sich z. B. um die Lemmata Lapsus memoriae (S. 290) und Emplastrum (S. 174). Die Lexeme aus dem Französischen betragen etwa $14 \%$ beim Buchstaben L (z. B. Lettre de marque, S. 293) und etwa $25 \%$ beim E (z. B. Eau de Lavande, S. 166). Bei diesen Lemmata überwiegen nichtadaptierte Lexeme, aus denen viele zu den Fachgebieten militärisch und kaufmännisch gezählt werden. Mehrere Lexeme stammen auch aus dem Griechischen (etwa $8 \%$ beim L und $22 \%$ beim E). Im Wörterbuchabschnitt L findet man auch eine kleine Anzahl von Lemmata aus dem Italienischen, Englischen, Niederländischen, Spanischen und aus dem Sanskrit. Beim E handelt es sich um das Türkische, Hebräische, Arabische oder Niederdeutsche.

Die Lemmata, die in Sommers NWVW zu finden sind, erscheinen auch in den meisten zur Analyse verwendeten Wörterbüchern aus dem 19. Jh. Im Falle, dass die Anzahl von Bedeutungen bei einem Lemma mit den Bedeutungsangaben in anderen Wörterbüchern nicht übereinstimmt, sind es vorwiegend die anderen Nachschlagewerke, in denen mehrere Bedeutungsangaben vorkommen. Diese Wörterbücher führen sehr oft auch mehrere Angaben zu Lemmata an. Es handelt sich z. B. um die Aussprache, die Angabe der Wortart oder der Etymologie.

Das letzte Kriterium betrifft das Vorkommen von Lemmata in Wörterbüchern von Adelung und Grimm. Aus den Lexemen, die Sommer in sein NWVW aufnahm, erscheinen bei Adelung und Grimm nur sehr wenige von ihnen. Bei Grimm beträgt der Prozentanteil etwa $11 \%$ beim Buchstaben L und ungefähr $3 \%$ beim E. Im zweitgenannten Wörterbuch findet man etwa $9 \%$ der Lemmata auf L und etwa $4 \%$ beim Buchstaben E. ${ }^{20}$ Das Ergebnis entspricht der Tatsache, dass es sich im Falle von Grimms und Adelungs Wörterbuch um keine Verdeutschungs- oder Fremdwörterbücher handelt, und dass diese Autoren daher vorwiegend längst etablierte Lexeme in ihre Werke aufnahmen.

20 Im Rahmen einiger Wörterbuchartikel dienen in Adelungs Wörterbuch weitere Lexeme als Sublemmata. 


\section{Sommers und Campes Verdeutschungsarbeit im Vergleich}

Dem vorhergehenden Abschnitt, der sich lediglich mit Sommers Werk befasst hat, folgt abschließend ein kurzer Vergleich ${ }^{21}$ seiner Arbeit mit dem wohl bekanntesten Verdeutschungswörterbuch von Campe. Sommers Arbeit wird auf diese Weise nicht mehr isoliert betrachtet, sondern in den Kontext der damaligen puristischen Bestrebungen eingeordnet.

Es zeigt sich, dass die Idee, ein puristisches Wörterbuch zu verfassen, nicht nur bei Sommer, sondern auch bei anderen Autoren eng mit ihren politischen Anschauungen zusammenhängt. Während sich aber Sommers Beschäftigung mit dem Purismus durch den Widerstand gegen Napoleon und Franzosen erklären lässt, war Campes politische Motivation einer anderen, aufklärerischen Art. Sein Ziel bestand darin, Lexeme fremden Ursprungs in der deutschen Sprache zu ersetzen, damit auch Menschen aus niederen Gesellschaftsschichten einen Zugang zur Bildung haben. Diese können dann nach der Veränderung des absolutistischen Systems streben (vgl. Schiewe 1988: S. 34, 190f.). Campes Bestrebungen wurden im zunehmenden Alter immer radikaler, was einige Gelehrte zur Kritik seiner Arbeit veranlasste (vgl. Schiewe 1988: 141 und Lemmata Purist und Purismus in Grimm). Sommers Äußerungen zufolge (s. 2. 2.) ist er wohl auch zu radikaler gesinnten Sprachpuristen zu rechnen. Bei ihm steht jedoch eine Untersuchung, die auf diesen Aspekt in seinen lexikographischen Werken eingehen würde, noch aus.

Die Adressatengruppe, auf die sich ihre Werke richten, ist bei beiden Lexikographen sehr ähnlich, obwohl sich Sommers Äußerungen widersprechen (s. 2. 2.). Trotz dieses Widerspruchs ist die intendierte Leserschaft offensichtlich unter dem Bürgertum zu finden, das auch von Campe bevorzugt wurde..$^{22}$ Obwohl sich die Autoren in ihrer Forderung nach eher kürzeren Verdeutschungen einig sind (vgl. Schiewe 1988) ${ }^{23}$, unterscheiden sich ihre Programme in dem Umfang. Sommers kurzes Verzeichnis von Verdeutschungsregeln, die er seinen Lesern vorlegt, kann sich mit Campes durchgedachtem und komplexem Verdeutschungsprogramm, das den Adressaten im Vorspann präsentiert wird, kaum messen (vgl. Schiewe 1988, Orgeldinger 1999).

Nicht nur der Umfang ihrer Programme, sondern auch die Struktur von Wörterbuchartikeln sieht in den beiden Werken unterschiedlich aus. Sommers Artikeln mit nur wenigen zusätzlichen Informationen, kurzen Beispielen und ggf. den Angaben zum Fachgebiet stehen Campes längere Artikel gegenüber, in denen er eine längere Reihe von Verdeutschungen angibt und dann bewertet. Beispiele stammen aus bekannten Werken, aus denen er manchmal längere Ausschnitte zitiert. In einigen Wörterbuchartikeln wird auch die Aussprache der zu verdeutschenden nichtindigenen Lexeme angegeben (vgl. Campe 1801).

21 Ein detaillierter Vergleich wird v. a. dadurch erschwert, dass Sommer zu vielen Problemen keine Stellungnahme abgab.

22 Anders ging z. B. Johann Christoph Adelung in seiner Arbeit vor, bei der er sich auf die Welt der Adligen konzentrierte (vgl. Haß-Zumkehr 2001: S. 107).

23 Manche von Campes Verdeutschungen waren jedoch sehr komplex (vgl. Schiewe 1988). 
Wie der kurze Vergleich zeigt, bedeuten die Überschneidungen in den Verdeutschungsprogrammen und anderen Umständen nicht automatisch, dass die Konzeptionen von Wörterbüchern sehr ähnlich bzw. identisch aussehen werden, sondern sie können auch sehr unterschiedlich ausfallen. Sommers Werk ähnelt viel mehr anderen zeitgenössischen Nachschlagewerken, während Campe seinen eigenen Weg ging.

\section{Schlussfolgerungen und Ausblick}

Das NWVW von Johann Gottfried Sommer stellt eines der vielen Werke dar, die sich im 19. Jahrhundert mit dem Sprachpurismus befassten. Mit der Veröffentlichung seines Wörterbuchs, das wohl wegen seiner Herausgabe außerhalb des zentralen deutschen Sprachraums heutzutage weniger bekannt ist, wollte Sommer seinen Beitrag zu puristischen Bestrebungen seiner Zeit leisten. Obwohl er keine theoretischen Schriften über Sprachpurismus verfasste, konnten aus dem Metateil seines Wörterbuchs und aus der Analyse zweier Wörterbuchabschnitte einige Grundregeln seiner Verdeutschungsarbeit und auch Informationen über seine Wörterbuchkonzeption gewonnen werden.

Obgleich Sommers Verdeutschungsarbeit durch seine politischen Ansichten, v. a. seine negative Stellungnahme gegen Franzosen motiviert wurde, ergab sich aus der Analyse, dass die meisten Lemmata lateinischer Herkunft sind. Der Prozentanteil von französischen Lemmata ist viel geringer. Trotz seines Vorhabens blieb er also der auch in anderen Nachschlagewerken vorkommenden Verteilung der Lemmata treu. Die Tatsache, dass die meisten Lemmata adaptiert wurden, zeigt, dass diese Ausdrücke in der deutschen Sprache bereits längere Zeit benutzt und in irgendeiner Weise an das deutsche Sprachsystem angepasst wurden. Auch aus dem Vorkommen der Komposita, die indigene und fremde Elemente kombinieren, kann geschlossen werden, dass einige Entlehnungen sogar zur Bildung neuer Wörter verwendet wurden. Die meisten Lexeme sind Autosemantika (mehr als $70 \%$ ), es handelt sich vorwiegend um Substantive, Verben und Adjektive. Den Großteil der Lemmata bilden Simplizia (um 70 \%). Zu den am häufigsten vorkommenden Fachgebieten in analysierten Abschnitten gehören (in der Klassifizierung des Autors) die Bereiche Arzneikunde, juristisch, militärisch und mythologisch. Im Gegensatz zu Sommers Werk erscheinen bei Grimm und Adelung nur sehr wenige in NWVW vorkommende Lemmata. Bei Grimm handelt es sich um etwa $3 \%$ beim E und $11 \%$ beim L und im Falle von Adelung sind es etwa $4 \%$ beim Buchstaben E und $9 \%$ beim L. Die meisten Lemmata wurden aber in anderen Wörterbüchern aus dem 19. Jh. belegt.

Aus der Tatsache, dass bei der Analyse viele andere Wörterbücher, u. a. auch Fremdwörterbücher, verwendet wurden, die ähnliche Konzeption wie Sommers Wörterbuch zu verfolgen scheinen, aber nicht als Verdeutschungswörterbücher bezeichnet werden, ergibt sich die Frage nach dem Unterschied zwischen Verdeutschungswörterbüchern und Fremdwörterbüchern. Die Verdeutschungswörterbücher können für eine Unterkategorie von Fremdwörterbüchern oder für einen selbständigen Wörterbuchtyp gehalten werden. ${ }^{24}$

24 Kirkness (1990) behauptet am Anfang seines Aufsatzes (S. 1170), dass Verdeutschungswörterbücher einen 
In Verdeutschungswörterbüchern spielen die Verdeutschungen eine wichtige Rolle, die als Ersatz von Wörtern fremden Ursprungs dienen sollen. Die Fremdwörterbücher dienen wiederum nicht oder nicht primär der Verdeutschung und Vermeidung von Fremdwörtern in der Zielsprache und manchmal vermitteln sie den Benutzern das enzyklopädische Wissen. Bezüglich der unterschiedlichen Ziele, die diese Werke verfolgen, sollte es sich folglich um unterschiedliche Wörterbuchtypen handeln.

Was die Konzeption von Verdeutschungswörterbüchern betrifft, sind sie nach Kirkness (1990: 1173) durch kurze Artikel gekennzeichnet, was Sommers Konzeption entspricht, aber es gibt auch solche Fremdwörterbücher, in denen kurze Artikel zu finden sind $^{25}$, und umgekehrt befinden sich in einigen Verdeutschungswörterbüchern längere Artikel, z. B. im Wörterbuch von Campe. Dieses Kriterium genügt also allein nicht, um ein Werk als Verdeutschungswörterbuch zu bezeichnen.

Da manche Wörterbücher zweifellos beide Typen kombinieren oder nach ihrem Titel zu kombinieren glauben (s. unten), muss auch die dritte Kategorie unterschieden werden, deren Vertreter an der Schwelle zwischen Verdeutschungswörterbüchern und Fremdwörterbüchern stehen. Der Analyse zufolge wird das NWVW von Sommer zu dieser Kategorie gezählt. Einerseits lassen sich in diesem Werk kurze Artikel finden, andererseits befinden sich im Wörterbuch auch Artikel, deren Ziel nicht in der Verdeutschung des Lemmas, sondern in der Erklärung des Lexems und in der Belehrung des Benutzers besteht.

Allerdings erhebt sich die Frage, welche Rolle der vom Autor gewählte Wörterbuchtitel spielt, d. h. ob ein Wörterbuch, das in seinem Titel als Verdeutschungswörterbuch bezeichnet wird, auch automatisch zu den Verdeutschungswörterbüchern mit entsprechender Konzeption gezählt werden kann, oder ob die Bezeichnung eines solchen Wörterbuchs lediglich von der Mode oder politischen Ansichten seines Autors abhängt. Ebenfalls lässt sich erst anhand weiterer Untersuchungen feststellen, ob es überhaupt einige Werke gibt, die nur Verdeutschungen beinhalten (d. h. Verdeutschungswörterbücher im engeren Sinne) und ob es daher möglich ist, eine präzise Unterscheidung zwischen Fremdwörterbüchern, Verdeutschungswörterbüchern und den Werken, die an der Schwelle zwischen ihnen stehen, vorzunehmen.

\section{Quellen- und Literaturverzeichnis}

Adami, Wilhelm (1842): Fremdwörterbuch, in welchem alle im gewöhnlichen Leben vorkommenden Fremdwörter erklärt werden [online]. Grünberg - Leipzig: Verlag von W. Lewysohn, unter: https:/ $/$ books.google.cz/books?id=VPtIAAAAcAAJ\&printsec $=$ frontcover\&hl $=$ cs\#v $=$ onepage \&q $\& \mathrm{f}=$ false [02.11.2019].

Adelung, Johann Christoph (1793-1801): Grammatisch-kritisches Wörterbuch der Hochdeutschen Mundart [online]. Leipzig, unter: http://woerterbuchnetz.de/Adelung/ [02.11.2019].

Typ von Fremdwörterbüchern darstellen. An einer anderen Stelle des Textes (S. 1173 f.) stellt er die beiden Wörterbuchtypen gegenüber und führt einige Unterschiede von ihnen an.

25 Es handelt sich z. B. um das Fremdwörterbuch, in welchem alle im gewöhnlichen Leben vorkommenden Fremdwörter erklärt werden (1842) von Wilhelm Adami und Ein teutscher Dictionarius (1571) von Simon Roth. 
BLKO = Biographisches Lexikon des Kaiserthums Oesterreich, Bd. 35: Sinacher-Sonnenthal [online]. Wien 1877: die k. k. Hof- und Staatsdruckerei, unter: http://www.literature.at/viewer.alo ?objid=11783\&page $=294 \&$ scale $=3.33 \&$ viewmode $=$ fullscreen $[02.11 .2019]$.

Campe, Joachim Heinrich (1801): Wörterbuch zur Erklärung und Verdeutschung der unserer Sprache aufgedrungenen fremden Ausdrücke. Ein Ergänzungsband zu Adelungs Wörterbuche, Bd. 1 [online]. Braunschweig: Schulbuchhandlung, unter: https://books.google.cz/books?id= U41CAQAAIAAJ\&printsec $=$ frontcover\&hl $=\mathrm{cs} \# \mathrm{v}=$ onepage\&q\&f=false [02.11.2019].

Deutsches Wörterbuch. Gütersloh: Bertelsmann-Lexikon-Verlag 1997.

Duden, Fremdwörterbuch. Mannheim - Wien - Zürich: Dudenverlag 1997.

Eisenberg, Peter (2011): Das Fremdwort im Deutschen. Berlin - New York: Walter de Gruyter.

Fremdwörterbuch. Leipzig: Bibliographisches Publikum Leipzig 1966.

Grimm, Jacob - Grimm, Wilhelm u. a. (1854-1961): Deutsches Wörterbuch von Jacob Grimm und Wilhelm Grimm [online]. Leipzig, unter: http://woerterbuchnetz.de/DWB/ [02.11.2019].

Haß-Zumkehr, Ulrike (2001): Deutsche Wörterbücher - Brennpunkt von Sprach- und Kulturgeschichte. Berlin - New York: Walter de Gruyter.

Kirkness, Alan (1990): Das Fremdwörterbuch. In: Hausmann, Franz Josef - Reichmann, Oskar Wiegand, Herbert Ernst - Zgusta, Ladislav (Hrsg.): Wörterbuch. Ein internationales Handbuch zur Lexikographie. 2. Teilband. Berlin - New York: Walter de Gruyter, S. 1168-1178.

Kirkness, Alan (1998): Das Phänomen des Purismus in der Geschichte des Deutschen. In: Besch, Werner - Betten, Anne - Reichmann, Oskar - Sonderegger, Stefan (Hrsg.): Sprachgeschichte: ein Handbuch zur Geschichte der deutschen Sprache und ihrer Erforschung. 1. Teilband. Berlin - New York: Walter de Gruyter, S. 407-416.

Müller, Helmut - Krieger, Karl Friedrich - Vollrath, Hanna (1995): Nakladatelství Lidové noviny. Praha.

NND = Neuer Nekrolog der Deutschen, Bd. 1 [online]. Weimar 1851, unter: http://reader.digitalesammlungen.de/de/fs1/object/display/bsb10070890_00113.html [12.04.2018].

Orgeldinger, Sibylle (1999): Standardisierung und Purismus bei Joachim Heinrich Campe. Berlin - New York: Walter de Gruyter.

Polenz, Peter von (1994): Deutsche Sprachgeschichte vom Spätmittelalter bis zur Gegenwart. Band II: 17. und 18. Jahrhundert. Berlin - New York: Walter de Gruyter.

Roth, Simon (1571): Ein teutscher Dictionarius [online]. Augspurg, unter: https://ia902608. us.archive.org/11/items/bub_gb_Cg9NAAAAcAAJ/bub_gb_Cg9NAAAAcAAJ.pdf [02.11.2019].

Schiewe, Jürgen (1988): Sprachpurismus und Emanzipation. Joachim Heinrich Campes Verdeutschungsprogramm als Voraussetzung für Gesellschaftsveränderungen. Hildesheim - Zürich New York: Georg Olms Verlag.

Sommer, Johann Gottfried (1819): Neuestes wort- und sacherklärendes Verteutschungswörterbuch aller jener aus fremden Sprachen entlehnten Wörter, Ausdrücke und Redensarten, welche die Teutschen bis jetzt, in Schriften und Büchern sowohl als in der Umgangssprache, noch immer für unentbehrlich und unersetzlich gehalten haben; verbunden mit einer Erklärung auch der weniger bekannten Kunstwörter und andern Ausdrücke der teutschen Sprache. Ein höchstnützliches Handbuch für Geschäftsmänner, Zeitungsleser, und alle gebildete Menschen überhaupt [online]. Prag, unter: https://books.google.cz/books?id=Sn9cAAAAcAAJ\&printsec $=$ frontcover\&hl=cs\#v=onepage\&q\&f=false [02.11.2019].

Straßner, Erich (1995): Deutsche Sprachkultur. Von der Barbarensprache zur Weltsprache. Tübingen: Max Niemeyer Verlag. 
Mgr. Eva Janečková / eva.janeckova01@upol.cz

Univerzita Palackého v Olomouci, Filozofická fakulta, Katedra germanistiky Křřžkovského 10, 77180 Olomouc, CZ

This work can be used in accordance with the Creative Commons BY-SA 4.0 International license terms and conditions (https://creativecommons.org/licenses/by-sa/4.0/legalcode). This does not apply to works or elements (such as image or photographs) that are used in the work under a contractual license or exception or limitation to relevant rights 
